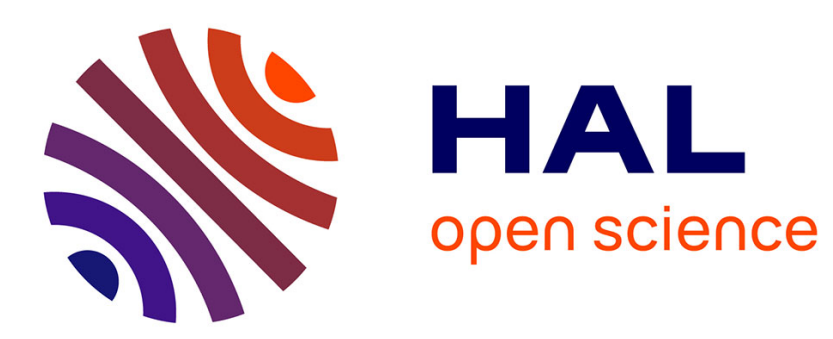

\title{
Étudier la contestation en contexte autoritaire : le cas cubain
}

\author{
Marie Laure Geoffray
}

\section{To cite this version:}

Marie Laure Geoffray. Étudier la contestation en contexte autoritaire : le cas cubain. Politix, 2011, 93 (1), 10.3917/pox.093.0029 . halshs-01726557

\section{HAL Id: halshs-01726557 https://shs.hal.science/halshs-01726557}

Submitted on 8 Mar 2018

HAL is a multi-disciplinary open access archive for the deposit and dissemination of scientific research documents, whether they are published or not. The documents may come from teaching and research institutions in France or abroad, or from public or private research centers.
L'archive ouverte pluridisciplinaire HAL, est destinée au dépôt et à la diffusion de documents scientifiques de niveau recherche, publiés ou non, émanant des établissements d'enseignement et de recherche français ou étrangers, des laboratoires publics ou privés. 


\title{
ÉTUDIER LA CONTESTATION EN CONTEXTE AUTORITAIRE : LE CAS CUBAIN
}

\author{
Marie Laure Geoffray
}

De Boeck Université I Politix

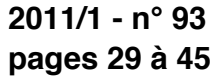

ISSN 0295-2319

Article disponible en ligne à l'adresse:

http://www.cairn.info/revue-politix-2011-1-page-29.htm

Pour citer cet article :

Geoffray Marie Laure , «Étudier la contestation en contexte autoritaire : le cas cubain »,

Politix, 2011/1 n93, p. 29-45.

Distribution électronique Cairn.info pour De Boeck Université.

@ De Boeck Université. Tous droits réservés pour tous pays.

La reproduction ou représentation de cet article, notamment par photocopie, n'est autorisée que dans les limites des conditions générales d'utilisation du site ou, le cas échéant, des conditions générales de la licence souscrite par votre établissement. Toute autre reproduction ou représentation, en tout ou partie, sous quelque forme et de quelque manière que ce soit, est interdite sauf accord préalable et écrit de l'éditeur, en dehors des cas prévus par la législation en vigueur en France. II est précisé que son stockage dans une base de données est également interdit. 


\title{
Étudier la contestation en contexte autoritaire : le cas cubain
}

\author{
Marie Laure Geoffray
}

\begin{abstract}
Résumé - Cet article interroge la possibilité d'observer la contestation en contexte autoritaire. À partir d'une enquête de onze mois sur le terrain cubain, il se propose d'y apporter trois éléments de réponse. Tout d'abord, une méthode d'enquête ethnographique, bien qu'elle doive faire l'objet d'une réflexivité particulière afin de ne pas réifier la contrainte du contexte, permet de dépasser l'opposition souvent instituée entre État autoritaire et mouvances contestataires. Ensuite, le choix de sites d'observation originaux - ici le rapport des enquêtés à l'espace et leur sens de la dramaturgie - offre des pistes d'analyse pertinentes pour saisir le sens et les effets de modes de contestation non discursifs dans un contexte qui criminalise la parole dissidente. Enfin, plutôt que de tenter d'évaluer la politisation des acteurs, cet article se propose d'analyser comment le politique émerge en situation.
\end{abstract}


$\mathrm{T}$ ôt, le 17 décembre 2006, je rejoins l'atelier du collectif d'artistes Omni Zona Franca, à Alamar, petite ville périphérique située à quinze kilomètres de La Havane, afin de participer à leur pèlerinage annuel au Rincón de Saint Lazare ${ }^{1}$, saint de la santé et de la guérison. Dans l'aube, les artistes, torses nus, se couvrent le visage et le corps d'inscriptions : "Pour la santé de la poésie ", "Offrande aux énergies occultes du peuple ", ou encore "Pour la liberté de l'esprit ». Une fois prêts, nous sortons dans la rue et arraisonnons un bus qui passait par là. La scansion de textes poétiques résonne de toutes parts, comme en écho. On entend des éclats de rire, des salutations au fur et à mesure que d'autres rejoignent le groupe, en cours de trajet. Un slogan est psalmodié à l'envi : « Pour la santé de la poésie, que s'ouvrent les chemins ». Malgré l'heure matinale, les passagers du bus sortent de leur torpeur. Certains ponctuent une lecture d'un rire ou commentent l'apparence des artistes. Beaucoup semblent les observer avec méfiance. Une question revient : qu'avait la poésie à faire avec le pèlerinage au Rincón ? Les artistes répondent invariablement : comme la santé, elle est nécessaire à la vie. Arrivé au terminus du bus, le cortège est rejoint par d'autres pèlerins-poètes et, dans un joyeux tumulte, tous se mettent en route sur l'avenue menant au Rincón.

Ce pèlerinage ne s'apparente pas à une mobilisation, telle qu'on en connaît en contexte démocratique. Pourtant, les artistes investissent la rue avec une visibilité travaillée dans un contexte dans lequel l'usage collectif de slogans, hors des manifestations officielles, est connoté comme une prise de parole dissidente. En même temps, les artistes ne créent pas l'événement, puisqu'ils investissent un pèlerinage religieux autorisé. Les slogans sont peints sur les visages et sur les corps, non sur des pancartes, et la revendication d'une liberté poétique dégonfle la tension occasionnée par la charge contestataire du premier terme. Comment dès lors appréhender ces modes d'action collective non conventionnels, fondés sur la pratique artistique du détournement et de la transgression?

Le choix d'une situation autoritaire amène à déplacer partiellement la focale proposée par ce dossier : l'observation de mobilisations. À Cuba, les manifestations de rue sont orchestrées par les organisations de masse para-étatiques qui encadrent la population. S'il existe quelques défilés d'opposants politiques, ceux-ci ont un impact local faible du fait de leur répression systématique par les autorités. Ce travail s'intéresse donc à d'autres pratiques contestataires, qui n'empruntent pas au répertoire classique des mobilisations, mais ne sont pas non plus interprétables en termes de résistance. Il s'agit de festivals de rap ou de poésie, d'ateliers de réflexion et d'actions directes ou performances dans des lieux publics.

1. Le Rincón (littéralement le Coin) de Saint Lazare est une petite église, située en grande banlieue de La Havane. Le 17 décembre, des milliers de fidèles convergent vers ce lieu afin de demander à Saint Lazare, qui fait l'objet d'une dévotion particulière à Cuba, santé et guérison. 
J'ai, pour ce faire, mené une enquête de type ethnographique ${ }^{2}$ auprès de la nébuleuse de collectifs contestataires à La Havane, croissante depuis les années 2000, à la faveur de l'autonomisation partielle du champ culturel. Informels au départ, ces collectifs ont été partiellement intégrés dans des institutions culturelles locales ou sectorielles, chargées de les canaliser. J'en ai choisi trois, parce qu'ils existent depuis plus d'une décennie (continuité dans le temps), ont un turnover relativement faible de leurs membres (continuité dans la trajectoire des acteurs), une existence officielle (malgré des rapports conflictuels avec les institutions) et sont actifs dans les marges du champ culturel tout en investissant certains espaces politiques. Leur répertoire est donc hybride au carrefour de la création culturelle et de l'action collective. Il s'agit de la mouvance hip-hop, d'Omni Zona Franca (Omni ZF), groupe de poètes, plasticiens et performeurs, et d'un collectif de jeunes enseignants, chercheurs et promoteurs culturels réunis sous le nom de Cátedra - Chaire - Haydée Santamaria.

Quoique souvent présentés comme « marginaux » socialement, car résidents de quartiers populaires ou périphériques de La Havane, la plupart des membres des collectifs sont, en réalité, issus de familles révolutionnaires qui ont bénéficié à plein des réformes sociales des années 1960 et 1970 (accès à l'éducation, mobilité sociale). S'ils contestent aujourd'hui l'ordre social et politique institué par la Révolution, ils sont aussi les héritiers de l'idéal de souveraineté nationale et de justice sociale porté par les révolutionnaires historiques, ce qui explique leur positionnement hétérodoxe, mais non dissident vis-à-vis du régime.

À partir de l'enquête réalisée auprès de ces collectifs, cet article propose trois éclairages pour répondre à une même question : comment étudier la contestation en contexte autoritaire? Il montre en premier lieu qu'une enquête d'inspiration ethnographique est particulièrement appropriée pour dépasser la tendance à la réification d'entités comme l'État et la société civile. Il s'attache ensuite à étudier le sens et les effets de pratiques contestataires non conventionnelles à partir de deux stratégies d'observation : le rapport des enquêtés à l'espace et la dimension performative de leurs modes d'action. Enfin, l'article propose de déplacer la focale de la question de la politisation vers celle des enjeux posés par les activités de catégorisation (politique/artistique) effectuées par les collectifs et par les autorités.

2. Cinq terrains, d'une durée totale de près d'un an, ont été effectués entre 2005 et 2010. Cependant, il n'a pas toujours été possible de conduire une enquête ethnographique idéale dans la continuité et la densité, comme le serait un long terrain ininterrompu par des contingences extérieures au temps de la recherche. Il est donc plus juste de caractériser la démarche suivie d'enquête d'inspiration ethnographique. 


\section{Ethnographier la contestation en contexte autoritaire}

Contrairement à d'autres contextes autoritaires, il n'est pas faisable de travailler, sur le long terme, avec les milieux d'opposition, comme en témoigne l'expulsion du territoire cubain d'une étudiante française après moins d'un mois d'enquête sur ce sujet, au printemps 2009. Conduire une enquête ethnographique sur la contestation à Cuba requiert donc le choix d'un autre objet que les mouvements d'opposition. En même temps, le déplacement induit mène à une focalisation sur des formes de contestation non moins pertinentes, dans la mesure où elles permettent d'effectuer un retour réflexif sur l'appréhension de la contestation en contexte autoritaire.

\section{Transformer une contrainte d'enquête en une stratégie de recherche}

Bien que la pertinence de la typification de régimes soit désormais interrogée $^{3}$, les contextes autoritaires demeurent encore trop souvent théorisés comme des lieux d'exercice d'un pouvoir vertical et fondé sur la répression. Cette théorisation n'est pas sans conséquences. Elle entraîne souvent une surinterprétation, en termes de résistance, de l'analyse des activités déviantes dans ces contextes et une focalisation sur les mouvements dissidents. Dans le cas cubain, la difficulté d'accès au terrain conduit les chercheurs qui s'intéressent à l'émergence de mouvances critiques à travailler sur la documentation disponible et à se focaliser sur les acteurs visibles depuis l'étranger, autrement dit les opposants politiques qui ont développé une stratégie de communication tournée vers l'international. Cette méthode d'enquête pose deux problèmes.

Le premier problème est d'ordre pratique. J'ai pu vérifier à plusieurs reprises que les sources alternatives d'information, produites par des journalistes dits indépendants, n'étaient pas toujours fiables. Or celles-ci sont souvent utilisées telles quelles dans de nombreux travaux sur l'opposition cubaine. Le second problème est d'ordre analytique. La reprise à leur compte, par les chercheurs, des catégories indigènes d'appréhension de la réalité mobilisées par les dissidents (État/société civile, répression/résistance etc.), tend à réifier les modes de compréhension de la société cubaine et à surévaluer le rôle des dissidents, envisagés d'un point de vue normatif comme les nécessaires vecteurs d'une démocratisation du régime.

Une enquête ethnographique, au sens d'« observation prolongée [...] d'un milieu, de situations et d'activités ${ }^{4}$ ", permet d'échapper à ces écueils car elle éclaire ce qui se joue à l'intérieur d'un régime qui continue à se reproduire plus de vingt ans après sa chute annoncée. Cette démarche méthodologique autorise

3. Dabène (O.), Geisser (V.), Massardier (G.), dir., Autoritarismes démocratiques, démocraties autoritaires, Paris, La Découverte, 2008 ; Camau (M.), Massardier (G.), dir., Démocraties et autoritarismes : fragmentation et hybridation des régimes, Paris, Karthala, 2009.

4. Cefaï (D.), dir., L'engagement ethnographique, Paris, Éd. de l'EHESS, 2010, p. 7 (l'auteur souligne). 
en effet le repérage de collectifs contestataires moins visibles depuis l'étranger que la dissidence, mais tout aussi organisés et plus actifs dans des arènes publiques locales, moins réprimés donc plus observables, notamment grâce aux dynamiques d'interaction qu'ils génèrent avec des publics et avec les autorités.

Cette démarche ne va cependant pas de soi. L'entrée sur le terrain est rendue difficile par les démarches bureaucratiques nécessaires pour obtenir un permis de séjour et l'immersion dans le quotidien des enquêtés se heurte souvent à de fortes réticences de leur part. Quand l'accès au terrain est finalement sécurisé, l'intensité du partage, avec les enquêtés, de sentiments comme la peur ou la paranoïa, génère un rapport de forte empathie envers eux, ce qui nécessite un retour réflexif long sur l'expérience du terrain.

\section{Le partage de la contrainte de l'incertitude. De l'expérience subjective à la réflexivité analytique}

S'il n'est jamais évident, quel que soit le contexte, de négocier une présence continue au sein d'un collectif, qu'il s'agisse d'une organisation ou d'un regroupement plus flou d'individus, cette négociation est particulièrement contrainte à Cuba. En effet, l'existence - réelle ou supposée - d'agents « espions » agissant pour le compte du gouvernement, au sein de la population, normalise fortement les discours et les comportements. Cette incertitude permanente sur l'identité réelle des interlocuteurs au quotidien engendre une méfiance structurelle envers tout enquêteur, même - et parfois surtout - s'il est de nationalité étrangère ${ }^{5}$. Cette méfiance s'est traduite, lors de mes premiers mois de terrain, par une véritable cannibalisation de mon temps d'enquête : j'étais invitée à participer à des réunions qui n'avaient pas lieu ; j'obtenais des promesses d'entretien non tenues; $j$ 'accourais à de nombreux rendez-vous où mes interlocuteurs ne se présentaient pas...

Quand des relations de confiance se sont finalement instaurées ${ }^{6}$, je me suis rendue compte que j'avais été contaminée par cette incertitude permanente. Consciente de la précarité de mon accès au terrain cubain, dépendant de l'octroi de visas universitaires à la convenance des autorités ${ }^{7}$, et n'ayant pas déclaré le véritable sujet de mon enquête, je me surpris à adopter les mêmes attitudes que mes enquêtés, comme le fait de baisser la voix - réflexe généralisé - quand

\footnotetext{
5. Marc Perry, chercheur américain, décrit comment il a été accusé par un producteur de rap cubain, en pleine réunion entre rappeurs et Panthères noires, d'être un agent de la CIA. Cf. Perry (M.), Rap, Race and Social Transformation in Contemporary Cuba, thèse pour le doctorat de sociologie, Université du Texas, Austin, 2004. 6. Sur le mode de la ruse de l'enquêteur qui doit « donner son temps, sans compter » pour compenser partiellement le « vol » de l'intimité de l'enquêté, et sa mise à profit par l'enquêteur pour obtenir une reconnaissance et une légitimité professionnelles, cf. Schwartz (O.), Le monde privé des ouvriers, Paris, PUF, 1990, p. 52-53.

7. La possibilité d'enquêter sur le terrain cubain est liée à l'obtention d'un visa universitaire. Ce type de visa ne s'obtient que par l'intermédiaire du soutien d'une institution universitaire sur place. Or il n'est pas accordé facilement ni même régulièrement (on peut l'obtenir une fois puis se le voir refuser).
} 
la conversation prenait un tour politique. De peur de ne pouvoir terminer mon enquête, mon travail de terrain a été contaminé par le sentiment de devoir calculer mes faits et gestes dans un certain clair-obscur, lié à mon appréhension subjective de ce que je pouvais ou non faire, de ce qui serait ou non toléré par les autorités. Ces sentiments de peur, de paranoïa et parfois de colère face à l'incertitude et à la fragilité ressenties ont profondément marqué mon appréhension du contexte cubain. Pour les tenir à distance, au moment de restituer l'expérience du terrain, il m'a fallu les relier à ma position d'enquêtrice étrangère.

L'expérience nouvelle que constituait pour moi le fait de calculer et d'agir hors d'un État de droit m'ont longtemps conduite à survaloriser l'impact de la contrainte de l'incertitude pour évaluer les frontières du possible et du jouable. Mon expérience intime de celle-ci m'avait en effet paru insupportablement anxiogène et je l'avais considérée comme inhibante pour les activités des collectifs. Si cela avait bien été le cas lors de leur émergence, dans une période de fermeture politique marquée, à la fin des années 1990, je ne compris que tardivement que cette contrainte avait en partie été routinisée par les enquêtés, régulée par leur insertion dans des institutions officielles et par la mise en place de relations de jeu fondées sur une coopération et une semi-confiance avec certains responsables culturels ou politiques, et compensée par leur sentiment (et leur affirmation publique) de ne jamais œuvrer contre la Révolution. Si la peur en tant qu'expérience sociale demeure donc importante pour comprendre certains choix des collectifs - comme celui, en amont de ma recherche, d'un ancrage institutionnel - elle est aujourd'hui moins centrale que l'existence d'une conflictualité négociée avec les autorités.

Plutôt que d'envisager la contrainte du contexte comme un donné, ce retour réflexif sur la pratique du terrain amène donc à étudier comment le contexte contraint l'action des collectifs, tout en l'habilitant à travers les prises qu'il offre au jeu et au détournement.

\section{De l'observation à l'interprétation des pratiques : une réflexion sur la performativité en acte}

Afin de mieux saisir ce qui fait le nœud de la contestation, dans le contexte autoritaire cubain, $\mathrm{j}$ 'ai choisi deux stratégies d'observation originales. Je me suis intéressée à la mise en espace et à la dramaturgie des activités des collectifs. J'entends la notion de dramaturgie comme une composition dramatique, une mise en scène publique pour et avec des publics ${ }^{8}$. J'appelle performances dramaturgiques les actions des collectifs qui constituent des mises en espace ou des mises en scène publiques qui contestent l'ordre social et politique local. L'étude de cette dramaturgie nous renseigne sur les contraintes, notamment spatiales,

8. Cefaï (D.), «Les cadres de l'action collective. Définitions et problèmes », in Trom (D.), Cefaï (D.), dir., Les formes de l'action collective, Paris, Éd. de l'EHESS, 2001. 
qui pèsent sur la contestation, ainsi que sur certains modes d'interaction avec les autorités, dont les effets peuvent s'avérer performatifs.

\section{Performances dramaturgiques}

J'ai choisi de contraster ici deux performances d'Omni Zona Franca dans deux espaces urbains distincts : une action d'appropriation spatiale et de détournement des normes prégnantes en centre-ville; et la dramatisation d'une situation locale problématique en périphérie.

La première performance a lieu en pleine rue 23 , la rue centrale très passante sur laquelle se trouvent des cinémas, des théâtres, des hôtels huppés, le centre de presse international et le fameux glacier Coppelia, toujours bondé. Carrefour urbain, lieu de rencontre et de passage, de déambulations touristiques, à proximité d'institutions prestigieuses et de lieux de pouvoir, la rue 23 est toujours quadrillée de policiers en uniforme ou en civil et de fonctionnaires chargés d'effectuer des contrôles divers. Choisir la rue 23 pour y organiser une performance signifie donc s'exposer : agir devant un public large et diversifié, mais aussi prendre un risque du fait du prix accordé à cet espace urbain et du contrôle dont il fait l'objet.

En ligne, les uns derrière les autres, une quinzaine de membres d'Omni ZF ont remonté la rue 23, en marchant en arrière et au milieu de la chaussée, entre les deux files des voitures qui s'y croisaient. Objets de l'attention des passants et des conducteurs interloqués, mais aussi hilares (certains sifflaient, d'autres applaudissaient, des conducteurs klaxonnaient, l'ordre de la queue du glacier Coppelia se rompit), les membres d'Omni ZF furent arrêtés dans leur marche par quelques policiers. Ceux-ci les admonestèrent en invoquant l'interdiction de manifester sur la voie publique. Les performeurs répondirent qu'il ne s'agissait en aucun cas d'une manifestation, mais d'une action artistique. N'ayant pas d'ordre pour arrêter une activité de cette sorte, les policiers, après avoir rapidement délibéré entre eux, permirent la reprise de la marche qui continua pendant encore plusieurs pâtés de maison avant que les artistes ne décident eux-mêmes de s'arrêter et de rentrer à Alamar.

Cette performance, loufoque au premier abord, est en fait significative. Alors que le centre ville constitue un espace particulièrement contrôlé, balisé et normé ${ }^{9}$, des performeurs résidant en périphérie s'y sont rendus pour transgresser les usages socialement admis de cet espace. Ils ont proposé une relecture de la marche dans l'espace urbain, en occupant la chaussée réservée aux

9. Le paysage urbain havanais est parsemé de slogans révolutionnaires peints à même les murs ou sur de grands panneaux, à la fois dans la ville et le long de ses axes d'entrée et de sortie. Cf. l'analyse de Daniel Ortega, en termes d'inscription spatiale des normes, dans « En cada barrio: Timocracy, Panopticism and the Landscape of a Normalized Community », Culture Machine, 8, 2006. 
voitures, et en cheminant à l'envers, de dos, comme pour montrer qu'on peut renverser le regard porté sur les évidences les plus incorporées. Ils se sont ainsi appropriés, de façon provisoire, un lieu dont les jeunes Cubains sont de plus en plus soustraits par une politique de réhabilitation urbaine qui fait la part belle aux touristes. Ils ont enfin réussi à éviter d'être accusés de trouble à l'ordre public, en mettant en avant la dimension exclusivement artistique de leur action.

Il existe plusieurs niveaux de lecture de cette performance. Elle peut être interprétée comme une intervention purement graphique en situation, à la manière des happenings d'Allan Kaprow dans les années $1970^{10}$. En même temps, dans un contexte de normalisation particulièrement forte des territoires urbains, notamment en centre-ville, cette performance peut également être lue comme un test des frontières du possible. Alors que les espaces publics fonctionnent comme des espaces de "concurrence à la conformité ${ }^{11}$ ", les artistes s'approprient une portion de territoire urbain, le temps d'une performance, de manière pour le moins hétérodoxe. Ils créent « un effet de défamiliarisation, de décalage, de perturbation ${ }^{12}$ » qu'on peut interpréter comme l'expérimentation, avec et pour un public, d'une libération temporaire de certaines normes et de certaines contraintes. On peut donc qualifier cette performance de monstration publique de non-conformité ${ }^{13}$, au sens où elle entraîne une transgression des usages des espaces publics et permet l'expérimentation collective d'autres usages de ces espaces à travers une visibilité intentionnelle et travaillée.

La focalisation sur la mise en espace de la performance donne à voir comment les performeurs s'approprient un territoire normé et incitent leurs publics au détournement de ces normes. Cependant, l'appropriation de l'espace n'est qu'éphémère, dans un lieu hautement contrôlé. Et la qualité également éphémère du public rassemblé ne permet pas d'évaluer la portée concrète de l'action. C'est pourquoi j'ai souhaité la contraster avec un autre type de performance, dont les effets peuvent s'avérer performatifs.

10. Cf. Fluxus, Révolution : textes essentiels, Le Bouscat, L’Esprit du Temps, 2009. On y trouve des morceaux de textes d'Allan Kaprow qui distingue le happening des autres types de performance car dans le happening, le public est partie constitutive de la performance.

11. Autrement dit, des espaces dans lesquels les individus rivalisent de conformité discursive afin de "se laver ", de ne pas se faire " remarquer » et donc d'obtenir un crédit politique qui permet la continuité des activités économiques illégales. Cf. Bloch (V.), « Le sens de la lutte », Communisme, 85-86, 2006, p. 143.

12. Kellenberger (S.), "La mobilisation des artistes-activistes contre le néo-libéralisme », in Balasinski (J.), Mathieu (L.), dir., Art et contestation, Rennes, Presses de l’Université de Rennes, 2006, p. 196.

13. Je m'inspire ici de la formule de Charles Tilly qui définit un mouvement social comme repeated public display dans "From Interactions to Outcomes in Social Movements », in Giugni (M.), McAdam (D.), Tilly (C.), eds, How Social Movements Matter, Minneapolis, University of Minnesota Press, 1999. 


\section{Revendications performatives}

Je présente ici une action particulièrement significative d'un type d'intervention aux incidences concrètes ${ }^{14}$. Cette action a eu lieu à Alamar, ville de banlieue où Omni ZF dispose d'un atelier de travail, dans une galerie d'art qui dépend de la direction municipale de la culture. Elle avait pour objet l'amoncellement de déchets dans les rues de la ville, résultat de l'absence de collecte par les services de la municipalité, problème beaucoup moins prégnant dans les quartiers aisés ou centraux, stratégiques pour le tourisme.

L'action consiste à s'enfouir dans un tas d'ordures situé dans un endroit de passage, non loin d'un arrêt de bus très fréquenté, à l'heure où les habitants partent travailler, et de ne plus bouger. On est ici entre le die in (simuler la mort) et le zap (" action-éclair contre une cible déterminée ») ${ }^{15}$. Devant le tableau ainsi créé, une foule s'est rapidement assemblée avec les commentaires suivants : "Ils sont morts ? ", «Ils ont été assassinés ? ", « C’est de la provocation ? ». Certaines personnes dans l'attroupement, responsables de cellules locales d'organisations de masse alertèrent la police et les deux protagonistes de l'action furent amenés au commissariat local et gardés à vue.

Lissue de cette performance fut cependant favorable. Malgré l'intervention de la police, les deux artistes furent libérés rapidement, sur ordre de la plus haute instance du conseil municipal d'Alamar, grâce à l'intermédiation des autorités culturelles locales. Celles-ci se portèrent garantes de ces deux membres d'OMNI ZF sous leur tutelle, en avançant un argument difficilement contestable par les autorités politiques : la dimension avant-gardiste de l'art du collectif était le garant de leur intégrité " révolutionnaire ». Quant à la question de la collecte des déchets, elle fut déclarée prioritaire sur l'agenda du conseil municipal qui s'organisa pour trouver les financements nécessaires.

Pour comprendre les effets performatifs de cette dramatisation artistique d'une situation quotidienne problématique, il faut revenir sur plusieurs éléments : la dimension fortement transgressive de l'action, contrebalancée par le soutien populaire et celui des autorités locales, et la question de l'interprétation politique d'un mode d'action artistique.

Les articles 53 et 54 de la Constitution cubaine restreignent fortement les droits des citoyens, en matière de liberté d'association et d'expression ${ }^{16}$. La for-

\footnotetext{
14. Il s'agit d'une action à laquelle je n'ai pas pu assister car elle a eu lieu en 2001. J'ai cependant pu reconstituer sa dramaturgie à travers les récits de quatre membres d'Omni ZF et les photos qu'ils prirent de l'action, les conversations menées avec des habitants du quartier qui avaient été des témoins directs du scandale produit sur la voie publique et de discussions informelles avec deux responsables des affaires culturelles à la municipalité d'Alamar.

15. Broqua (C.), «Sida et stratégies de représentation. Dialogues entre l'art et l'activisme aux États-Unis », in Balasinski (J.), Mathieu (L.), dir., Art et contestation, op. cit.

16. L'article 53 " accorde aux citoyens la liberté d'expression et de la presse, conformément aux fins de la société socialiste », grâce à la " propriété étatique ou sociale » des moyens de communication de masse,
} 
mulation de critiques peut cependant être tolérée, si elle a lieu à l'intérieur des institutions, sans publicité, et que des solutions aux problèmes soulevés sont négociées directement avec les autorités ${ }^{17}$. C'est ce qu'un dicton populaire cubain résume ainsi : bajo techo todo, en la calle nada (sous un toit tout, dans la rue rien). À l'inverse, toute exposition publique d'un problème est dénoncée par les autorités comme une atteinte à la souveraineté de l'État. Les autorités appellent cela « faire le jeu de l'ennemi » : donner des arguments aux « ennemis de la Révolution » pour critiquer le régime et le délégitimer. Performer dans la rue, en prenant pour cible des sujets sensibles comme les problèmes matériels, exponentiels depuis la crise économique engendrée par la dissolution de l'URSS, constitue donc une transgression de ces lois.

Linsertion locale d'Omni ZF à Alamar, banlieue sinistrée par la crise économique et isolée par la pénurie de transports publics, permet cependant de comprendre le calcul des risques effectué par le collectif et la possibilité d'une réception non répressive de son action. Dans un espace enclavé comme Alamar où les rumeurs circulent vite, leur réputation collective, en tant que "bande de fous ", est déjà bien ancrée. En effet, la faible offre culturelle de la ville tranche avec leur organisation d'activités hautement visibles car polémiques. Or ces activités sont organisées dans un cadre institutionnel puisque Omni ZF travaille dans un atelier de travail, octroyé par la direction municipale de la culture, dans ses propres locaux. Les membres du collectif doivent en échange négocier la mise en œuvre de leurs projets, quoiqu'ils passent souvent outre. Ces fonctionnaires locaux soutiennent, malgré tout, les activités du collectif, car leur inscription dans leur programmation leur permet d'obtenir un rayonnement culturel qui dépasse les frontières d'Alamar et d'acquérir un certain prestige dans leurs fonctions. Il existe donc des relations d'interdépendance, quoique conflictuelles, entre le collectif et sa tutelle locale, dont le décryptage est essentiel pour comprendre le calcul des risques effectué par le collectif et les effets performatifs de la performance décrite.

Le choix par les artistes de la cible de l'action est lié à l'existence d'un terreau favorable. Ils s'attaquent en effet à un problème concret et durable dans la ville, longuement évoqué par ses habitants, quoique sans résultat, lors des réunions des Comités de Défense de la Révolution $(\mathrm{CDR})^{18}$, ce qui ne peut que susciter l'adhésion du public. Ensuite, la performance se déroule en l'absence

\footnotetext{
censée garantir « leur usage au service exclusif du peuple travailleur et de l'intérêt de la société ». L'article 54 stipule que les citoyens disposent du droit d'association, de manifestation et de réunion « dans le cadre des organisations de masse ».

17. Hoffmann (B.), «Voice via Web. The Changing Dynamics of Civil Society in Pre- and Post-Internet Cuba ", communication au colloque de l'ECPR, joint sessions of workshops, Lisbonne, 14-18 avril 2009.

18. Les CDR sont des comités de quartier aux fonctions politiques (surveillance des comportements), sociale (campagnes de vaccination, aide sociale aux familles défavorisées), de communication (explication et discussion des orientations du gouvernement) et de gestion (problèmes quotidiens du quartier).
} 
de toute revendication discursive. Son interprétation est donc polysémique, ce qui octroie aux autorités culturelles locales des armes pour la défendre face aux autorités politiques.

J'insiste ici sur cette polysémie car, si l'intention des protagonistes était certes la dramatisation d'une situation sanitaire intenable, la performativité de leur action tient, comme le théorisait Yurchak à propos des actes ritualisés de discours en URSS, à sa production d'effets imprévus ${ }^{19}$. À partir des lectures de Bourdieu et Derrida de l'œuvre de John Austin, auteur du célèbre « dire c'est faire $^{20}$ ", Yurchak combine une analyse des « manifestations symboliques » liées à l'énonciation, en partie contenues " dans les conditions institutionnelles de leur production et de leur réception » (Bourdieu) ${ }^{21}$ mais également actives dans le « pouvoir sémiotique » du discours (Derrida) ${ }^{22}$ et donc productrices d'effets non anticipés liés à la rencontre entre une parole et un contexte, autrement dit la génération de sens nouveaux en situation. C’est aussi ce que soutient Judith Butler, pour qui le performatif fait événement, à partir du moment où il génère un sens non anticipé23.

Dans le cas présenté ici, c'est l'imbrication entre l'avant-garde de la forme artistique (le happening) et l'avant-garde du mode de revendication politique (interpellation publique en situation), quasi inexistant à Cuba, qui produit des effets inédits. Le sens de la performance dramaturgique étudiée n'était pas a priori intelligible, et en aucun cas univoque. Ce sont les habitants de la zone qui l'interprétèrent comme une action de revendication contre l'absence de collecte des déchets. Cette interprétation faisait sens pour eux, car elle faisait écho à leur perception de l'inefficacité des autorités locales. La circulation de cette interprétation causa le scandale public et lança une polémique hors les murs des organisations officielles. Si la réaction des habitants était une condition nécessaire à la performativité de l'action, elle n'était cependant pas suffisante. C'est la réponse des autorités devant ce mécontentement - trouver le financement nécessaire à la collecte - qui la rend véritablement performative. Sa réception par les destinataires qui comptent (ici les décideurs locaux), médiatisée par les réactions des habitants, est ce qui la constitue en une action à portée politique. Au-delà des intentions des protagonistes, le sens politique de cette performance a donc été acquis en acte.

Il reste cependant un point aveugle dans cette analyse. Sans accès aux réunions internes ou à des comptes rendus du Parti Communiste Cubain (PCC), on ne peut que supputer les raisons pour lesquelles les autorités municipales

\footnotetext{
19. Yurchak (A.), Everything Was for Ever, Until it Was no More, Princeton, Princeton University Press, 2006, p. 19 et s.

20. Austin (J.), Quand dire, c'est faire, Paris, Seuil, 1970 [1 ìre éd. 1962].

21. Bourdieu (P.), «Le langage autorisé », Actes de la Recherche en Sciences Sociales, 5-6, 1975.

22. Derrida (J.), L'écriture et la différence, Paris, Seuil, 1967.

23. Butler (J.), Excitable Speech: A Politics of the Performative, New York, Routledge, 1997, p. 160 et s.
} 
d'Alamar décidèrent d'accéder aux demandes de la population à cette occasion, et non à d'autres. C'est d'ailleurs une des limites d'une enquête ethnographique : comment envisager le rôle de l'État sans en réifier les contours? Cette interrogation est d'autant plus forte dans un contexte contraint dans lequel l'information, et donc l'accès à de la documentation officielle, est fortement contrôlée.

Il me semble qu'une réponse à cette question se situe dans la construction d'une articulation entre une méthode ethnographique et une approche relationnelle du pouvoir ${ }^{24}$. Cette approche, qui consiste à définir le pouvoir en acte, permet en effet de l'envisager par sa dissémination, par son enracinement dans l'ensemble du corps social, et de restituer ainsi comment il s'exerce en venant contraindre la capacité d'action des sujets. Elle incite à prendre en compte la complexité de l'intrication entre la part de jeu et de résistance et celle d'acceptation et parfois d'adhésion, certes ambiguë et ambivalente, des dominés vis-à-vis de cette emprise du pouvoir. L'articulation de cette approche avec une méthode d'enquête ethnographique est particulièrement fructueuse car elle conduit à étudier des situations localisées, des agencements et des configurations de pouvoir. Dans les deux performances étudiées ici, c'est l'insertion d'une séquence d'activité dans un contexte d'interactions existant en amont - l'existence d'une normalisation spatiale particulièrement forte ou bien l'ancrage institutionnel des collectifs - et sa réception, donc ses effets en aval - qui permet de comprendre ce qui se joue, en termes à la fois pratiques et cognitifs, dans certains modes d'action des collectifs.

La pertinence de cette articulation tient finalement à ce qu'elle permet d'envisager de manière conjointe la domination et sa contestation. D'un côté, elle facilite une compréhension plus fine de la possible performativité de modes d'action contestataires. De l'autre, elle offre une appréhension plus complexe de l'État cubain par la mise en évidence de l'existence d'échelles et de secteurs différenciés à l'intérieur de l'appareil politique.

\section{Questionner les frontières du politique}

Cette dernière partie propose de revenir sur la question de la politisation des modes d'action contestataires dans un contexte autoritaire. Elle se fonde sur l'hypothèse selon laquelle il est moins pertinent de tenter d'évaluer la politisation des acteurs que de repérer les ambiguités et les contradictions de leur rapport au politique. J'ai en effet pu observer, à l'instar de Nina Eliasoph sur un

\footnotetext{
24. Je me réfère aux travaux de Bayart (J.-F.), notamment son article fondateur : «L'énonciation du politique ", Revue française de science politique, 35 (3), 1985, ainsi qu'à Weber (M.), Économie et société, Paris, Plon 1971 (sur le diptyque ordre/docilité) et à Foucault (M.), Sécurité, Territoire, Population. Cours au collège de France 1977-1978, Paris, Gallimard-Seuil, 2004.
} 
autre terrain ${ }^{25}$, l'existence d'une prolifération de régimes d'expression et de justification chez les mêmes acteurs, selon la situation d'énonciation et d'interlocution. Non seulement cette pluralité discursive est à analyser en tant que telle, mais elle invite à déplacer la focale vers les enjeux de catégorisation (politique/ artistique) des activités des collectifs, car celle-ci délimite, par contrecoup, l'espace de jeu dont les acteurs disposent.

\section{Un rapport ambigu au politique}

Tout au long de mon enquête, j'ai pu constater l'ambiguïté du rapport au politique des membres des collectifs. Cet extrait d'entretien avec José (Omni ZF) est particulièrement éloquent à cet égard.

«-C’est quoi pour toi la Révolution? Être révolutionnaire?

- [...] C'est une question qui revient. Patrycja [Sartora, réalisatrice du documentaire Alamar Express sur Omni ZF] par exemple voulait faire un documentaire politique sur nous. Mais on lui a dit que cela ne nous intéressait pas, c'est des trucs qui ne nous intéressent pas. »

Il ajoute plus tard :

«Le travail d'Omni n'est pas toujours politique mais c'est important que tout le monde se charge de politique comme dans les sociétés primitives. À Cuba, c'est difficile de penser de manière apolitique.»

Dans les faits, une partie importante des enquêtés qui ont, en entretien, refusé toute caractérisation de leurs activités comme politiques, ont souvent tenu, loin du micro de mon enregistreur, des propos contradictoires. Les usages du terme " politique » varient selon le contexte social et l'environnement physique, mais aussi selon le contexte sémantique immédiat, comme en témoigne l'exemple de José. Il met à distance le politique quand il identifie une de mes questions comme trop orientée, mais le réintroduit dans la conversation après avoir proposé tout un développement sur la transformation morale individuelle que devrait véritablement signifier " être révolutionnaire ». Il refuse donc l'imposition d'un sens politique à son action, mais n'hésite pas à employer le terme de son propre chef. Cet exemple typique est un signe de la volonté des acteurs de soigneusement contrôler le discours qu'ils émettent. Ces apparentes contradictions ne signifient pas un rejet univoque du politique. Elles doivent être comprises à l'aune des cadres cognitifs qui enserrent l'appréhension de la réalité sociale à Cuba.

En langage indigène, faire de la politique signifie être militant, donc membre du PCC, ou dissident. De façon singulièrement contre-intuitive, les activités de

25. Eliasoph (N.), Avoiding Politics: How Americans Produce Apathy in Everyday Life, Cambridge, Cambridge University Press, 1998, p. 15-16. 
ces deux types d'acteurs ne sont d'ailleurs pas séparées dans l'ordre de l'expérience. Les enquêtés évoquaient souvent les activités des opposants comme de la « haute politique »(alta política), celle des autorités, des transactions et des collusions, donc comme la face inverse de la politique gouvernementale, et à ce titre similaire. Face à l'injonction officielle à la conformité politique, la dissidence ne représentait donc pas pour eux une alternative aux effets de saturation engendrés par la politisation totale de la vie sociale ${ }^{26}$.

Outre la connotation négative du terme de «politique », organiser des activités considérées comme telles, ne serait-ce que par ses voisins, constitue une prise de risque, du fait de la généralisation de la pratique de la dénonciation. Des décennies de socialisation aux normes révolutionnaires ont en effet enseigné aux citoyens que les seuls canaux d'expression politique légitimes sont les réunions et activités des organisations de masse et du PCC. Toute activité non officielle et surtout organisée avec des codes proches de ceux des manifestations officielles (défilé, usage de panneaux et slogans) est nécessairement contrerévolutionnaire, dans les cadres cognitifs de la population cubaine. Par ailleurs, et là aussi de façon contre-intuitive, la notion de « droits humains » ne constitue en aucun cas un slogan mobilisateur. Elle a, comme la notion de " politique ", acquis une connotation négative, car elle est depuis longtemps associée, dans les discours officiels, au lobby anticastriste de Miami et à la CIA comme bras armé de la politique étrangère américaine. Face à cette contrainte, les enquêtés tentent de limiter les risques potentiellement engendrés par la conflictualité de leurs modes d'action en balisant les cadres de réception de leurs activités, non seulement par les autorités, mais aussi par leurs publics.

\section{Les enjeux de catégorisation des activités contestataires}

Cet extrait d'entretien avec Adriana (Omni ZF) témoigne des enjeux de présentation des collectifs ainsi que du travail de catégorisation de leurs activités.

«On nous critiquait. On parlait de prostitution et on nous disait que ça n'existait pas ici, mais nous on en parlait de façon poétique. On parlait aussi de la désertification. Et des gens ont demandé ce qu'était ce groupuscule de droits des humains qui faisait ça... C'est monté jusqu’à Abel Prieto [ministre de la Culture]. Un jour, on a fait une performance avec un panneau. Ernesto portait le panneau et les gens avaient peur de le lire. À cause de ça, Ernesto a dû aller chez les flics. Car à l'époque, c'était politique de sortir dans la rue avec un panneau. On nous dénigrait, des gens disaient qu'on avait fait une manif. Les gens pensent pas, ils reproduisent ! Les vidéos de la performance ont été confisquées et montrées à Abel Prieto. Mais il a dit que c'était de l'art. Il est plus intelligent,

\footnotetext{
26. Les autorités cubaines classent comme « politiques » de nombreux délits comme les tentatives de sortie illégales (sans l'autorisation de sortie nécessaire) du pays, puisqu'elles estiment que cela porte atteinte à la Révolution. C'est ce dont témoigne la récente libération de prisonniers dits politiques par les autorités (et non par les ONG comme Amnesty International), car ayant commis ce type de délit.
} 
plus ouvert. Et on a continué. On disait des choses qui ne se disaient pas. Et les gens s'habituaient, ils se disaient : "Ah ce sont les fous là !” ".

L'analyse de cet extrait d'entretien permet de comprendre que les enquêtés revendiquent une pratique de transgression de certaines frontières, mais tentent d'en imposer une lecture exclusivement artistique. Ainsi, le sujet tabou que constitue la prostitution sur le plan politique est légitimé par son appréhension poétique. Cet extrait montre de plus que le collectif travaille à naturaliser comme artistique l'usage de codes perçus comme relevant du seul champ politique. Les artistes peignent, par exemple, des slogans poétiques à même la peau et portent des panneaux sur le corps comme des hommes-sandwichs, plutôt qu'à bout de bras comme dans une manifestation. Dans le cas décrit par Adriana, la pancarte en question appelait les habitants d'Alamar à prendre soin de leur environnement (pour la santé de l'écologie).

La réception, par les autorités, de cette entreprise de naturalisation de codes associés au champ politique légitime n'est pas uniforme. Dans le cas de la performance évoquée ci-dessous, les autorités politiques locales et une partie des habitants avaient dénoncé cette apparition publique comme politique. Pourtant, au haut niveau de décision politique dans la sphère culturelle, le ministre Abel Prieto fit le choix d'entériner l'autocatégorisation du collectif : happening artistique en faveur de l'écologie. La catégorisation d'une même action peut donc varier avec l'échelle d'autorité et la sphère de compétence. Ce choix ne doit cependant pas être compris comme la volonté de légitimer une fois pour toutes les activités du collectif comme artistiques. Il s'agit plutôt de délimiter un espace de jeu, qui confere à la fois une certaine autonomie d'action au collectif, mais le rend tributaire d'un accord implicite avec la plus haute autorité culturelle. Tant que leurs activités restent formulées en termes artistiques, elles peuvent être tolérées.

Il faut par ailleurs remarquer que le choix de cette catégorisation, au plus haut niveau, des activités du collectif en termes d'" art » a entraîné un phénomène d'ajustement au niveau local. Dans les schèmes de perception des habitants, puisque ces activités ne sont pas réprimées, c'est qu'elles ne sont donc pas politiques. C'est ainsi qu'on peut interpréter la formulation d'Adriana : «À l'époque, c'était politique de sortir dans la rue avec un panneau ». Aujourd'hui donc, ça ne l'est plus, car les artistes ont gagné la bataille stratégique pour la définition de certaines de leurs activités. La naturalisation d'une expression contestataire publique par sa catégorisation officielle comme de l'art et sa répétition dans les mêmes lieux a en effet entraîné un déplacement de sens pour les habitants du quartier. Cela explique qu'ils aient recatégorisé les activités du collectif comme «folles ». Les membres du collectif sont depuis lors désignés, dans leur entourage, comme los locos de ahi (ces fous-là), caractérisation qui a entraîné une certaine routinisation locale de la portée transgressive de leurs activités, et leur a 
octroyé, de ce fait, des marges de manœuvre pour repousser, toujours plus loin, les limites du possible et du jouable.

Les frontières du politique restent cependant mouvantes. Les collectifs ne réussissent pas toujours à imposer leur propre définition de leurs activités, dont le sens peut se cristalliser en situation, comme je l'ai montré plus haut. C'est ce dont témoigne la censure subie par le rappeur Humbertico après le festival annuel de hip-hop en août 2003, pour avoir exhibé une pancarte sur laquelle on pouvait lire "plainte sociale »(denuncia social). Convoqué au commissariat le lendemain de son concert, il fut interdit de scène pendant quatre mois.

Humbertico avait, comme tous les rappeurs, passé le filtre de la censure préalable du festival et n'avait pas dissimulé l'usage qu'il ferait du panneau, dans une de ses chansons. En entretien, il m'a donc fait part de son incompréhension face à cette censure a posteriori, alors qu'il avait joué le jeu du dispositif de censure a priori. Humbertico sous-estime ici la construction d'un sens politique en situation à son usage du panneau. Selon les témoignages de journalistes internationaux qui couvraient l'événement, le public, particulièrement nombreux (plus de 3000 personnes) était surchauffé ce soir-là et réagit de manière démonstrative à la performance d'Humbertico, en chantant des slogans contre les policiers, ce qui créa de forts mouvements de foule. C'est donc dans l'interaction entre le rappeur et son public que l'usage du panneau, code emprunté au très fermé champ politique légitime, a pris une dimension performative, est soudain apparu comme politique aux yeux des autorités et a, par conséquent, justifié la censure du rappeur.

Les modes de catégorisation des activités des collectifs constituent donc un véritable enjeu, puisque leur continuité en dépend fortement. Tant qu'elles ne sont pas définies comme politiques par les autorités qui comptent - ici les autorités culturelles nationales, leur tutelle en dernier ressort - elles sont en grande partie protégées. Cette protection permet aux collectifs d'instituer des «structures de publicité ${ }^{27}$ » recevables et intelligibles pour leurs publics, et donc d'élargir leur espace d'action, puisque leurs activités deviennent alors socialement acceptables. Chez les autorités, la tolérance entretenue vis-à-vis des modes d'action des collectifs remplit une fonction politique : contribuer à incorporer ces nouvelles formes d'expression à la " fiction unanimiste ${ }^{28}$ » de la grande narration révolutionnaire. Tous jouent, dans les faits, sur la polysémie des termes (art, révolution...) et les malentendus qu'ils engendrent pour s'accorder a minima et assurer ainsi le maintien du jeu. Si les déplacements des frontières du politique, liés à la création d'effets de sens en situation, mettent en évidence que

27. Cefaï (D.), Pourquoi se mobilise-t-on ?, op. cit., p. 255.

28. Je reprends cette formule à Rigaud (F.), «Irak : l'impossible mouvement intérieur », in Bennani-Chraïbi (M.), Fillieule (O.), dir., Résistances et protestations dans les sociétés musulmanes, Paris, Presses de Sciences Po, 2003, p. 207. 
l'incertitude demeure un élément central de l'expérience des enquêtés, ce jeu a donc été partiellement routinisé. Il repose sur des accords implicites reconductibles entre autorités et collectifs.

\section{Conclusion}

Les différents éclairages proposés pour appréhender la contestation en contexte autoritaire permettent d'avancer quelques éléments de réflexion à plusieurs niveaux. Tout d'abord, une enquête de type ethnographique donne accès à des phénomènes contestataires non visibles ou non étudiables à distance. Cependant, cette méthode doit être particulièrement réflexive, dans un contexte autoritaire, afin de ne pas surévaluer certaines contraintes, vécues comme particulièrement fortes par l'enquêteur étranger en l'absence d'une socialisation comparable à celle des enquêtés. Ensuite, le choix de stratégies d'observation originales (rapport à l'espace, dramaturgie) des modes d'action des collectifs permet de montrer que l'intention contestataire investie dans les performances n'épuise pas le sens de ces actions. Celui-ci s'élabore en situation à travers la réception de ces actions par des publics et il s'avère variable selon les configurations d'acteurs et les relations d'interdépendance qui les unissent. Il est donc moins pertinent de chercher à repérer ce qui relèverait du politique dans les modes d'action des acteurs contestataires que d'observer leur entreprise de catégorisation de leurs activités comme exclusivement artistiques, sans cesser de travailler à naturaliser l'usage hétérodoxe de certains codes du champ politique légitime. Cette stratégie leur permet en effet de maintenir et parfois d'élargir leur espace de jeu vis-à-vis d'autorités aux positionnements variés selon les secteurs et les échelles de pouvoir.

Marie Laure Geoffray est docteure en science politique (IEP Paris, 2010) et postdoctorante dans le programme DesiguALdades du Lateinamerika-Institut (Freie Universität, Berlin). Sa thèse s'intitule :
Culture, politique et contestation à Cuba 1989-2009. Elle a notamment publié « Des intellectuels cubains après la chute du Mur ", Genèses, 77 (4), 2009.

mlgeoffray@gmail.com 